\title{
Crystal field coefficients for yttrium analogues of rare-earth/transition-metal magnets using density-functional theory in the projector-augmented wave formalism
}

\author{
Christopher E. Patrick and Julie B. Staunton \\ Department of Physics, University of Warwick, Coventry CV4 7AL, UK \\ E-mail: c.patrick.1@warwick.ac.uk
}

\begin{abstract}
We present a method of calculating crystal field coefficients of rareearth/transition-metal (RE-TM) magnets within density-functional theory (DFT). The principal idea of the method is to calculate the crystal field potential of the yttrium analogue ("Y-analogue") of the RE-TM magnet, i.e. the material where the lanthanide elements have been substituted with yttrium. The advantage of dealing with Y-analogues is that the methodological and conceptual difficulties associated with treating the highly-localized $4 f$ electrons in DFT are avoided, whilst the nominal valence electronic structure principally responsible for the crystal field is preserved. In order to correctly describe the crystal field potential in the core region of the atoms we use the projector-augmented wave formalism of DFT, which allows the reconstruction of the full charge density and electrostatic potential. The Y-analogue crystal field potentials are combined with radial $4 f$ charge densities obtained in selfinteraction-corrected calculations on the lanthanides to obtain crystal field coefficients. We demonstrate our method on a test set of 10 materials comprising 9 RE-TM magnets and elemental $\mathrm{Tb}$. We show that the calculated easy directions of magnetization agree with experimental observations, including a correct description of the anisotropy within the basal plane of $\mathrm{Tb}$ and $\mathrm{NdCo}_{5}$. We further show that the Y-analogue calculations generally agree quantitatively with previous calculations using the opencore approximation to treat the $4 f$ electrons, and argue that our simple approach may be useful for large-scale computational screening of new magnetic materials.
\end{abstract}

Submitted to: J. Phys.: Condens. Matter 


\section{Introduction}

Rare-earth/transition-metal (RE-TM) compounds, particularly those containing neodymium, samarium and dysprosium, are the highest performing permanent magnets on the commercial market [1, 2]. The key factor underpinning the success of these materials is the possibility of obtaining a huge magnetocrystalline anisotropy (MCA), i.e. a preferential direction for an object to be magnetized independent of its macroscopic shape [3], which originates from the highly-localized $4 f$ electrons of the lanthanide elements. Specifically, the unfilled shell of $4 f$ electrons forms a non-spherically symmetric charge cloud which sits in the (also non-spherically symmetric) crystal potential. The interaction between the $4 f$ cloud and the crystal potential, and the interaction between the spin and orbital degrees of freedom of the $4 f$ electrons themselves, results in a strong coupling of the RE magnetism to the crystal potential [4]. The spin-spin RE-TM interaction further couples the magnetic moments of RE to those of the transition metals iron or cobalt [5]. The TM provides a large saturation magnetization and high Curie temperature, which combine with the high MCA to form an excellent permanent magnet [6].

Experimental research into RE-TM permanent magnets has been carried out for over 50 years [7, 8, 9, 10, 11]. Computational research, particularly that based on parameter-free, "first-principles" methods, is a younger field by comparison [12]. However, the growth of computing power and a more widespread availability of modelling codes has led to a rapid increase in recent years of computational works aimed not only at understanding current RE-TM permanent magnets but also predicting the properties of new materials yet to be synthesized experimentally [13, 14, 15, 16, 17, 18, 19, 20, 21, 22, 23, 24, 25, 26. Here a first-principles approach is highly desirable, since the novel materials may require visiting a previously unexplored parameter space where the reliability of empirical models is unknown.

However, an enduring challenge presented by RE-TM magnets for first-principles calculations is how to simultaneously describe accurately the itinerant electrons of the TM and the highly-localized $4 f$ electrons of the lanthanide. Practical implementations of density-functional theory (DFT) [27], a first-principles methodology which is highly popular due to its versatility and accuracy, require approximating the exchangecorrelation (XC) contribution to the total energy of the electrons. Approximations based on the local spin density and the homogeneous electron gas (LSDA/GGA) work very well for itinerant electrons and form the basis of all widely-available DFT codes [28, 29]. Unfortunately, LSDA/GGA XC functionals do not describe the lanthanide elements well, with the $4 f$ electrons being too delocalized [30].

In a previous publication [31], we discussed some different approaches which attempt to correct the LSDA/GGA description of $4 f$ electrons. These approaches

include the "open core" scheme [32], which effectively removes the $4 f$ states from the valence band and freezes them in the core, dynamical mean-field theory (DMFT) [33], the self-interaction correction [34], and the LSDA/GGA $+U$ scheme [35]. In that 
publication we chose the (local) self-interaction correction (LSIC) [36] and combined it with the disordered local moment (DLM) picture of finite temperature magnetism [37] to calculate the magnetization and Curie temperatures of the entire series of RE-TM magnets with formula $\mathrm{RECo}_{5}$ [31]. However, using the same approach to calculate the MCA is problematic, because current LSIC and DLM implementations employ a spherical approximation for the potential at the RE site. The most important contribution to the MCA, i.e. from the crystal potential, is therefore incorrectly described 38].

If we wish to continue with our LSIC/DLM approach to obtain a comprehensive picture of RE-TM magnets, it is apparent that we must augment the original calculations to account for the non-spherical potential at the RE site. Fortunately, an entire theoretical framework has been developed to describe the effects of this asphericity, namely crystal field (CF) theory [39, 40, 41, 42]. Here, the potential at the RE site is expanded in terms of angular functions, and matrix elements with radial $4 f$ wavefunctions are quantified in terms of $\mathrm{CF}$ coefficients. Knowledge of the CF coefficients, combined with a description of the finite temperature TM magnetism, can lead to a very detailed picture of RE-TM magnetism [42].

The CF coefficients can be regarded as empirical parameters used to fit experimental data [43], but they can also be calculated. The seminal crystal field models were based on the potential set up by arrays of point charges [39, 40]. The advent of DFT allowed the CF coefficients to be calculated from first principles [12], originally from the electrostatic potential set up by the charge density [44, or later by also including the XC contribution [17, 45, 46, 47]. As usual, the $4 f$ electrons require special treatment, most frequently via the open core scheme [12]. Recent work has also seen the development of sophisticated techniques using DMFT to evaluate CF coefficients 20, or formulating CF theory in a Wannier basis [48].

One reason that a number of different approaches can be found in the literature is that CF theory is essentially empirical, and there is not a unique way of combining it with non-empirical DFT. In particular, in CF theory the $4 f$ electrons are spectators which feel the crystal field but do not themselves influence it [49]. But in a standard DFT calculation the CF potential contains contributions from all electrons, meaning that special treatment of the $4 f$ electron density (e.g. removing the non-spherical components [17]) is required.

Here, we go a step further and present a method where we completely remove the $4 f$ electrons from the crystal field, by calculating the CF potential of the yttrium (Y)-analogue of the RE-TM magnet. Here "Y-analogue" means that have we have replaced all lanthanide atoms with yttrium; for instance, the $\mathrm{Y}$-analogue of $\mathrm{Sm}_{2} \mathrm{Co}_{17}$ is $\mathrm{Y}_{2} \mathrm{Co}_{17}$. This approach is free from the complications of treating the $\mathrm{XC}$ energy of the $4 f$ electrons, and produces a potential which does not contain $4 f$ contributions.

Of course, the validity of the approach depends on whether the valence electrons of the lanthanide are well represented by Y. As a bare minimum, the lanthanide must be in a $3+$ state, so that the nominal valence configurations agree. For the widely 
used RE-TM magnets we believe this requirement to be well satisfied [12], but some care will be required in novel materials if the lanthanide is thought to undergo valence fluctuations [25] (for such materials, the open-core approximation would encounter the same problem as the Y-analogue model). In addition, our calculations on the $\mathrm{RE}^{3+} \mathrm{Co}_{5}$ compounds revealed a small contribution from $f$-type states around the Fermi level [31] which will be missing for the Y-analogue, as will be any effects due to the (spherically-symmetric) spin polarization of the $4 f$ electrons. Therefore the use of the Yanalogue must be considered an approximation. However, the advantage of making this approximation is then we are free to compute the potential using common LSDA/GGA functionals, using widely available DFT codes.

Our manuscript describes the implementation of the Y-analogue scheme, focusing particularly on two points. The first is that if the chosen DFT code is not an "allelectron" code, i.e. it does not treat core and valence electrons on the same footing, the charge density and potential will be incomplete in the core region of the atoms. By performing the calculations within the projector-augmented wave (PAW) formalism [50], as found in a number of popular codes including Quantum Espresso [51], GPAW [52] and the Vienna Ab initio Simulation Package (VASP) [53], it is possible to restore this core contribution as a post-processing step. In Secs. 2.3 2.6 we describe how this is done.

The second point is that in order to calculate CF coefficients, we require the radial distribution of the $4 f$ electrons. As we discuss in Section 2.7, here we use the spherically symmetric $4 f$ charge density obtained from a separate LSIC calculation. Therefore our method consists of two separate strands, calculated with different codes: one code provides the potential $V(\mathbf{r})$ for the Y-analogue and the other code provides the radial density $n_{4 f}^{0}(r)$ for the spherically-symmetric approximated RE-TM compound. Multiplying the two quantities and integrating yields the CF coefficients.

We have used our method to calculate CF coefficients for 10 materials, comprising 9 RE-TM magnets and elemental Tb. Where data is available we compare our calculations to previous work. The data demonstrate all of the correct qualitative trends with respect to experiment, and are in good quantitative agreement with open-core calculations. We therefore present the method as an approximate but simple scheme to calculate $\mathrm{CF}$ coefficients.

The rest of our manuscript is organized as follows. In Section 2 we start with a general overview of the crystal field picture and then discuss the specifics of obtaining the potential in the PAW formalism. We also discuss the calculation of the sphericallysymmetric $4 f$ density and some of the conventions regarding $\mathrm{CF}$ coefficients. In Section 3 we present the calculated CF coefficients and compare to data previously published. Finally in Section 4 we present our conclusions and discuss potential future developments. 


\section{Theory}

\subsection{Crystal field picture}

As comprehensively explained in Ref. [42] (and references therein), crystal field theory describes atomic-like electrons, which are eigenstates of a central potential and characterized by a set of quantum numbers $\left|L S J M_{J}\right\rangle$, perturbed by the CF potential $V(\mathbf{r})$. In the simplest case of a single magnetic sublattice subject to an external field $\mathbf{B}$ with a spin-orbit coupling quantified by $\lambda$, the CF Hamiltonian is [42]

$$
\hat{H}=\lambda \hat{\mathbf{L}} \cdot \hat{\mathbf{S}}+\mu_{B}(\hat{\mathbf{L}}+2 \hat{\mathbf{S}}) \cdot \mathbf{B}+\sum_{i} V\left(\mathbf{r}_{\mathbf{i}}\right) .
$$

Here $\mathbf{r}_{\mathbf{i}}$ denotes the position of a $4 f$ electron. $V(\mathbf{r})$ can be conveniently expanded in terms of angular functions centred on the RE site. Using the (complex) spherical harmonics $Y_{l m}(\hat{\mathbf{r}})$, this expansion is

$$
V(\mathbf{r})=\sum_{l m} V_{l m}(r) Y_{l m}(\hat{\mathbf{r}}) .
$$

Matrix elements of $\sum_{i} V\left(\mathbf{r}_{\mathbf{i}}\right)$ are products of radial and angular parts. The angular parts are rewritten and evaluated in terms of operators, and result in the appearance of Stevens coefficients $\alpha_{J}, \beta_{J}$ and $\gamma_{J}$, for $l=2,4,6$ respectively [54]. The radial part of the matrix element forms the CF coefficient, which is the quantity that we aim to calculate in this work:

$$
B_{l m}=\left(\frac{2 l+1}{4 \pi}\right)^{\frac{1}{2}} \int r^{2} n_{4 f}^{0}(r) V_{l m}(r) d r
$$

The sign has been defined such that a negative $V_{l m}(r)$ is attractive to an electron, which

is the opposite of a conventional electrostatic potential [42]. $n_{4 f}^{0}(r)$ is a sphericallysymmetric charge density associated with $4 f$ electrons which we discuss in Section 2.7 , while we discuss the $(2 l+1) /(4 \pi)$ prefactor in Section 2.8. First, we focus on calculating $V_{l m}(r)$.

\subsection{Kohn-Sham potential}

As mentioned in the Introduction, the original formulation of CF theory supposed the perturbing potential $V(\mathbf{r})$ to be electrostatic in origin [39]. However, in DFT the manybody system of interacting electrons is mapped onto non-interacting Kohn-Sham (KS) electrons, which experience both an electrostatic and an exchange-correlation potential. As discussed in Ref. [12], it is reasonable to include the $\mathrm{XC}$ contribution to the $\mathrm{CF}$ potential. However, since the $\mathrm{XC}$ potential is spin dependent one must either average over spins [12] or introduce spin-dependent CF coefficients [20]. To keep things general, we take the latter option and slightly modify (3) so that there is a dependence on spin $\sigma(=\uparrow, \downarrow)$ :

$$
B_{l m}^{\sigma}=\left(\frac{2 l+1}{4 \pi}\right)^{\frac{1}{2}} \int r^{2} n_{4 f}^{0}(r) V_{l m}^{\sigma}(r) d r
$$


where, inverting (2) and inserting the KS potential,

$$
\begin{aligned}
V_{l m}^{\sigma}(r) & =\int V_{\mathrm{KS}}^{\sigma}(\mathbf{r}) Y_{l m}^{*}(\hat{\mathbf{r}}) d \hat{\mathbf{r}} \\
& =\int\left[V_{\mathrm{H}}(\mathbf{r})+V_{\mathrm{XC}}^{\sigma}(\mathbf{r})\right] Y_{l m}^{*}(\hat{\mathbf{r}}) d \hat{\mathbf{r}} .
\end{aligned}
$$

In our approach $V_{\mathrm{KS}}^{\sigma}(\mathbf{r})$ is the self-consistent $\mathrm{KS}$ potential of the Y-analogue of the RETM magnet. Above we have split the KS potential into the electrostatic potential $V_{\mathrm{H}}(\mathbf{r})$ (which includes electrostatic electron-electron and electron-nuclear interactions), and the $\mathrm{XC}$ potential $V_{\mathrm{XC}}^{\sigma}(\mathbf{r})$.

\section{3. $P A W$}

In principle, if one performs an all-electron DFT calculation (particularly using atom-centred basis sets) then extracting $V_{l m}^{\sigma}(r)$ should be straightforward. However, calculations which make some distinction between core and valence electrons, e.g. those using pseudopotentials, do not deal directly with $V_{\mathrm{KS}}^{\sigma}(\mathbf{r})$ but rather with "pseudized" potentials and densities. The advantage of such schemes is that they remove the large computational effort required to describe the rapidly varying electronic wavefunctions in the core region [55].

The projector-augmented wave (PAW) formalism is a popular method to perform such calculations [50]. The full electronic+nuclear charge density is replaced with a pseudo-density $\tilde{\rho}(\mathbf{r})$, which has an associated pseudo-electrostatic potential which solves the Poisson equation $\nabla^{2} \tilde{V}_{\mathrm{H}}(\mathbf{r})=-4 \pi \tilde{\rho}(\mathbf{r})$ (Hartree atomic units). The difference between the full and pseudo-density is denoted $\Delta \rho(\mathbf{r})$. For each atom, an augmentation

region is defined, which is an atom-centred sphere with a radius of approximately 1-2.5 Bohr radii depending on the atom [56]. Crucially, outside the augmentation spheres the full and pseudo-densities are identical (i.e. $\Delta \rho(\mathbf{r})$ vanishes). Inside, the pseudodensity is constructed such that it has the same multipole moments as the full density. Therefore, the full and pseudo-electrostatic potentials also match each other outside the augmentation spheres [50, 52].

We thus write down a PAW version of (5):

$$
\begin{aligned}
V_{l m}^{\sigma}(r)= & \int\left[\tilde{V}_{\mathrm{H}}(\mathbf{r})+V_{\mathrm{XC}}^{\sigma}\left(n^{\uparrow}(\mathbf{r}), n^{\downarrow}(\mathbf{r})\right)\right] Y_{l m}^{*}(\hat{\mathbf{r}}) d \hat{\mathbf{r}} \\
& +\Delta V_{\mathrm{H} l m}(r) .
\end{aligned}
$$

$\Delta V_{\mathrm{H} l m}(r)$ is the angular-resolved correction to the electrostatic potential. Note that we have now also specialized to the case that the $\mathrm{XC}$ potential depends only on the spin densities at the point $\mathbf{r}$, i.e. the LSDA [28]. The all-electron spin-density is given by

$$
n^{\sigma}(\mathbf{r})=\tilde{n}^{\sigma}(\mathbf{r})+\sum_{l m} \Delta n_{l m}^{\sigma}(r) Y_{l m}(\mathbf{r}) .
$$

where $\tilde{n}^{\sigma}(\mathbf{r})$ is the electronic, spin-resolved contribution to the pseudo-density and $\Delta n_{l m}^{\sigma}(r)$ are the atom-centred corrections. 


\subsection{Angular expansions of pseudized quantities}

To obtain the angular expansions of the pseudo-electrostatic potential $\tilde{V}_{\mathrm{H} l m}(r)$ and the electronic pseudo-density $\tilde{n}_{l m}^{\sigma}(r)$, we use a Lebedev grid [57] of 5810 points on the unit sphere with vectors $\hat{\mathbf{r}}_{\mathbf{i}}$ and weights $w_{i}$. For example,

$$
\tilde{V}_{\mathrm{H} l m}(r)=4 \pi \sum_{i} w_{i} \tilde{V}_{\mathrm{H}}\left(\hat{\mathbf{r}}_{\mathbf{i}} r\right) Y_{l m}^{*}\left(\hat{\mathbf{r}}_{\mathbf{i}}\right) .
$$

PAW codes generally include a postprocessing option to output $\tilde{V}_{\mathrm{H}}(\mathbf{r})$ or $\tilde{n}^{\sigma}(\mathbf{r})$ on a grid, and the smoothness of the data allows one to obtain their values at arbitrary $\mathbf{r}$ through interpolation.

\subsection{Correction to the pseudo-electrostatic potential}

Recalling that $\Delta \rho(\mathbf{r})$ vanishes outside the augmentation sphere, the correction to the electrostatic potential is given by

$$
\Delta V_{\mathrm{H}}(\mathbf{r})=\int d \mathbf{r}^{\prime} \frac{\Delta \rho\left(\mathbf{r}^{\prime}\right)}{\left|\mathbf{r}-\mathbf{r}^{\prime}\right|}
$$

The angular expansion of $\Delta V_{\mathrm{H}}(\mathbf{r})$ is therefore

$$
\Delta V_{\mathrm{H} l m}(r)=\left(\frac{4 \pi}{2 l+1}\right) \int r^{\prime 2} d r^{\prime} \Delta \rho_{l m}\left(r^{\prime}\right) \frac{r_{<}^{l}}{r_{>}^{l+1}},
$$

with $r_{<}$and $r>$ respectively denoting the lesser and greater of $r^{\prime}$ and $r$.

\subsection{Correction to the pseudo-density $\Delta \rho_{l m}(r)$}

$\Delta \rho(\mathbf{r})$ consists of two contributions [52]. The first is from the nuclei, and requires replacing the soft "compensation charges" (which ensure the multipole moments of the full and pseudo-density agree [50]) with the point charge at the origin with a $-Z / r$ potential. The second contribution is the correction to the electron pseudo-density $\Delta n(\mathbf{r})$, which restores the rapid variation of the electron density close to the origin and also replaces the soft pseudo-core density with the contribution from the atomic-like core states. The resulting expression involves a number of PAW quantities and we give it in Appendix A. Here, we simply stress that all the quantities required are either already included in the PAW datasets or computed during the SCF calculation, so the computational effort required to obtain $\Delta \rho(\mathbf{r})$ is minimal.

As well as using $\Delta \rho(\mathbf{r})$ to calculate $\Delta V_{\mathrm{H}}(\mathbf{r})$, the spin-resolved all-electron density (7) allows the computation of the XC potential in the same angular representation. Thus, using the steps described above, Appendix A and (6), the angular resolved $\mathrm{CF}$ potential $V_{l m}^{\sigma}(r)$ appearing in (4) is obtained.

\subsection{The spherically-symmetric $4 f$ charge density}

We now consider the other ingredient required to calculate the CF coefficients, which is the spherically-symmetric $4 f$ charge density $n_{4 f}^{0}(r)$. In CF theory $n_{4 f}^{0}(r)$ originates 
from single-particle eigenfunction of the unperturbed central potential which enters the matrix element of the CF potential, $n_{4 f}^{0}(r)=\left|\psi_{4 f}(r)\right|^{2}$ [42], which is normalized as

$$
\int r^{2} n_{4 f}^{0}(r) d r=1
$$

Of course, the Y-analogue model used to obtain $V_{l m}^{\sigma}(r)$ does not provide $n_{4 f}^{0}(r)$, since the stated aim of the model was to remove the $4 f$ electrons. However, an approach which aligns closely to the CF picture is to perform an atomic-like calculation for a spherically-symmetric potential, where the potential corresponds to the RE atom embedded in the crystal. Here we describe such an approach, based on scattering theory and the local self-interaction correction (LSIC) [36].

The LSIC is an implementation of the SIC within the multiple-scattering, KorringaKohn-Rostoker (KKR) Green's function formalism of DFT [58]. In particular, the KS potential is by construction in "muffin tin" form, i.e. the potential is spherically symmetric within non-overlapping atom-centred spheres and surrounded by a flat potential interstitial region (it is also possible to use the "atomic sphere" construction, which removes the interstitial region and allows the overlap of spheres) [58]. Previously we used such calculations (which are done at the scalar-relativistic level) as starting points for fully-relativistic DLM calculations on RE-TM compounds for the entire $\mathrm{RECO}_{5}$ series from Y-Lu inclusive [31].

The scalar-relativistic potential obtained for the RE site from a self-consistent LSIC calculation can be inserted into the problem of the scattering of a free electron by an isolated, spherically-symmetric and finite-ranged potential. Apart from the regular and irregular solutions $Z$ and $J$, the central quantity in such a problem is the $t$-matrix [59]. The Green's function of the scattered electron is

$$
\begin{aligned}
G\left(\mathbf{r}, \mathbf{r}^{\prime}, E\right)= & Z(\mathbf{r}, E) t(E) Z^{\times}\left(\mathbf{r}^{\prime}, E\right) \\
& -Z(\mathbf{r}, E) J^{\times}\left(\mathbf{r}^{\prime}, E\right)
\end{aligned}
$$

where $\times$ denotes a left-hand solution to the radial equation, and matrix multiplication over angular indices has been implied (see Refs. [59, 60] for details). The single-particle density $n_{\mathrm{SP}}$ is obtained from the Green's function as

$$
n_{\mathrm{SP}}(\mathbf{r})=-\frac{1}{\pi} \operatorname{Tr} \int_{C} G(\mathbf{r}, \mathbf{r}, E) d E
$$

The contour $C$ is a rectangle in the complex plane which encloses the energies of the bound $4 f$ states, which in an LSIC calculation typically sit $\sim 1$ Ry below the Fermi level [31]. For the light lanthanides (i.e. atomic numbers smaller than $\mathrm{Gd}$ ) all of the bound $4 f$ states are included. For the heavy lanthanides only the states belonging to the unfilled spin subshell are enclosed in the contour, since these states are the ones responsible for the crystal field effects.

Since $Z$ and $J$ have the form of radial functions multiplied by spherical harmonics, it is quite straightforward to use $(12)$ and $(13)$ to extract the spherically-symmetric part of $n_{\mathrm{SP}}$. We then normalize this function to satisfy $(11)$ and set the result equal to $n_{4 f}^{0}(r)$, 


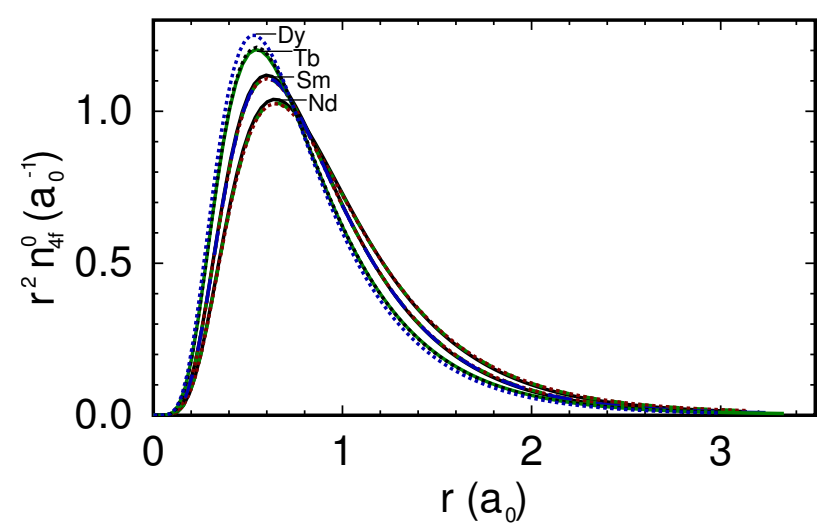

Figure 1. The spherically-symmetric $4 f$ electron density $n_{4 f}^{0}(r)$ calculated for 10 compounds: $\mathrm{NdCo}_{5}, \mathrm{SmCo}_{5}, \mathrm{~Tb}$ (solid lines); $\mathrm{NdFe}_{12}, \mathrm{SmFe}_{12}$ (dashed); $\mathrm{NdFe}_{12} \mathrm{~N}$, $\mathrm{SmFe}_{12} \mathrm{~N}, \mathrm{TbFe}_{2}, \mathrm{DyFe}_{2}$ (dotted); $\mathrm{Sm}_{2} \mathrm{Co}_{17}$ (long dash). The data fall onto four distinct curves depending on the lanthanide.

i.e.

$$
n_{4 f}^{0}(r)=\frac{\int n_{\mathrm{SP}}(\mathbf{r}) d \hat{\mathbf{r}}}{\int_{\Omega_{\mathrm{MT}}} n_{\mathrm{SP}}\left(\mathbf{r}^{\prime}\right) d \mathbf{r}^{\prime}} .
$$

The normalization in (14) has been performed within the muffin tin sphere $\Omega_{\mathrm{MT}}$. Accordingly, when calculating the CF coefficients from (4) the integral is also performed up to the muffin tin radius.

In Fig. 1 we plot $n_{4 f}^{0}(r)$ obtained for each of the 10 materials in our test set (more details regarding the test set can be found in Sec. 3.1). The most notable feature of Fig. 1 is that $n_{4 f}^{0}(r)$ depends on the lanthanide but not on the host compound. As a result, the 10 curves are effectively reduced to 4, corresponding to the number of lanthanides in the test set $(\mathrm{Nd}, \mathrm{Sm}, \mathrm{Tb}$ and $\mathrm{Dy})$.

Physically, the observation that $n_{4 f}^{0}(r)$ depends on the lanthanide but not the host compound is consistent with the picture of atomic-like $4 f$ electrons. Practically, this behaviour could be useful for studies screening a large number of candidate compounds based on their CF coefficients. To a good approximation, Fig. 1 supports the idea that one need not recalculate $n_{4 f}^{0}(r)$ for every compound, but rather predefine one function for each lanthanide to be used for the full set of candidates. Then the computational work would be restricted to calculating only the potential $V_{l m}^{\sigma}(r)$ for the Y-analogues. For the current manuscript, however, we have recalculated $n_{4 f}^{0}(r)$ for each compound.

\section{8. "B" and " $A$ " $C F$ coefficients and axis orientation}

Since we have chosen to expand the potential in terms of complex spherical harmonics (2), it is natural to work with the $\mathrm{CF}$ coefficients conventionally labelled $B$. These coefficients correspond to expanding the potential with Wybourne operators, which are related to the spherical harmonics by the prefactor appearing in (3) [41, 42]. Within this normalization one can distinguish "real" and "imaginary" CF coefficients depending 


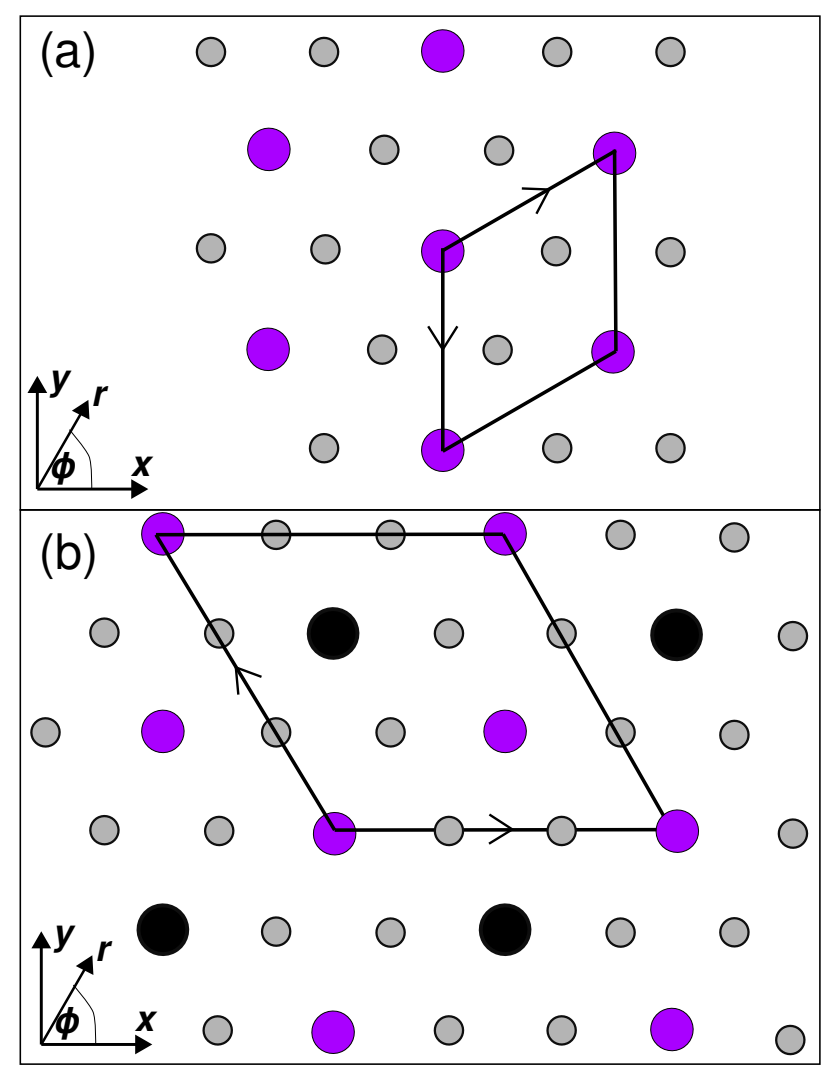

Figure 2. (a) The $\mathrm{RECo}_{5}\left(\mathrm{CaCu}_{5}\right)$ crystal structure [9], showing just the $a b$ plane containing the RE atom. The hexagonal unit cell according to the convention of Ref. 61] is shown with the cartesian axes and polar co-ordinate representation. (b) The $\mathrm{RE}_{2} \mathrm{Co}_{17}\left(\mathrm{Th}_{2} \mathrm{Zn}_{17}\right)$ crystal structure. Notice how the unit cell is rotated compared to (a), but the hexagonal array of Co atoms surrounding the RE are in the same orientation. Grey circles represent $\mathrm{Co}$, purple $\mathrm{RE}$ and black represents pairs of $\mathrm{Co}$ atoms (dumbbells).

on the relationship between $B_{l m}$ and $B_{l-m}$. For the materials considered here we have chosen the crystal axes such that the imaginary CF coefficients are zero, so $B_{l m}=B_{l-m}$.

In the past and present $\mathrm{CF}$ literature it is more common to find $\mathrm{CF}$ coefficients $\left[A_{l m}\left\langle r^{l}\right\rangle\right]$ which are based on Stevens operators [41]. Ref. [41] gives a table of factors $\alpha_{l m}$ which allow the conversion $\left[A_{l m}\left\langle r^{l}\right\rangle\right]=\alpha_{l m} B_{l m}$ to be performed. To facilitate comparison with previous work we perform this conversion and report our results using the Stevens form. We stress that despite the notation, $\left[A_{l m}\left\langle r^{l}\right\rangle\right]$ should be considered a single quantity in our calculations (hence the square brackets) and cannot be be factored into $A_{l m}$ and $\left\langle r^{l}\right\rangle$.

We also point out an issue relevant to materials with non-orthogonal axes, e.g. hexagonal crystals. In the expansion of (2), the conventional relation between polar and cartesian co-ordinates applies, i.e. $\hat{\mathbf{r}}=(\sin \theta \cos \phi, \sin \theta \sin \phi, \cos \theta)$. For cubic and tetragonal crystal structures, it makes sense to choose the crystal axes to coincide with the three cartesian directions. However, for a hexagonal system with the $c$ axis pointing in the $z$ direction, one can choose a crystal axis also to point in the $x$ or $y$ direction. 
The reason that such a choice has an impact on CF coefficients, specifically those with nonzero $m$, is illustrated in Fig. 2(a). Here the $\mathrm{SmCo}_{5}$ structure is shown, focusing on the $a b$ plane containing the Sm atoms. Figure 2(a) obeys the convention given in the book by Bradley and Cracknell [61], so that one of the hexagonal lattice vectors is parallel to the $-y$ direction. Such a system will in general have a nonzero $B_{66}$, which is also equal to $B_{6-6}$. However, one could construct a hexagonal co-ordinate system with a hexagonal vector parallel to the $x$ direction, corresponding to a $90^{\circ}$ rotation in the $a b$ plane. Since $Y_{6 \pm 6} \propto e^{ \pm 6 i \phi}, B_{66}$ and $B_{6-6}$ would flip sign with this choice of axes. Physically, the difference occurs because in one case a vector with $\phi=0$ and its origin at the RE site points towards the nearest neighbour Co atom, while for the other case the vector points exactly between two.

In this work, we mainly follow the convention of Fig. 2(a) when dealing with hexagonal systems. The key exception is $\mathrm{Sm}_{2} \mathrm{Co}_{17}$, where we have a hexagonal vector pointing along the $x$ direction [Fig. 2(b)]. This choice of axes for $\mathrm{Sm}_{2} \mathrm{Co}_{17}$ ensures that the imaginary $B$ coefficients are zero, and actually gives a local environment for the Sm atoms which is more similar to $\mathrm{SmCo}_{5}$ [i.e. nearest in-plane Co atoms lying close to the $\phi=0$ direction, which can be seen by comparing Figs. 2(a) and (b)].

We have drawn attention to this issue because not all codes agree on the cartesian orientation of hexagonal axes. For instance, the Hutsepot KKR code 62] used to perform the LSIC calculation uses the orientation of Fig. 2(a) for hexagonal crystals by default. However, in the Quantum Espresso package [51] or the Atomic Simulation Environment (ASE) 63] hexagonal axes are defined with a lattice vector pointing parallel to the $x$ direction [Fig. 2(b)]. With this in mind we think it useful when reporting crystal field coefficients with nonzero $m$ for hexagonal structures to also give the relationship between crystal axes and $\theta$ and $\phi$.

Finally, we note an additional potential source of confusion is that in experimental literature there are $B$ coefficients which are distinct from those introduced above. These alternative $B$ coefficients are the $\left[A_{l m}\left\langle r^{l}\right\rangle\right]$ quantities multiplied by the appropriate Stevens coefficient $\alpha_{J}, \beta_{J}$ or $\gamma_{J}$, depending on $l$ [64]. For clarity, we do not refer to these quantities any further here.

\subsection{Anisotropy constants from crystal field coefficients}

This work focuses on the calculation of CF coefficients. However, experimental studies involving magnetization measurements more commonly report anisotropy constants, which (for a uniaxial system) come from an expansion of the free energy in terms of the magnetization direction $\left(\theta_{0}, \phi_{0}\right)$ :

$$
\begin{aligned}
E\left(\theta_{0}, \phi_{0}\right)= & K_{1} \sin ^{2} \theta_{0}+K_{2} \sin ^{4} \theta_{0}+K_{3} \sin ^{6} \theta_{0} \\
& +K_{4} \sin ^{6} \theta_{0} \cos 6 \phi_{0} .
\end{aligned}
$$

For $\mathrm{Tb}$, where there is no contribution from the TM, we can calculate the $K_{i}$ values by diagonalizing the Hamiltonian in (1) within the ground $J$ manifold $(L=S=3$, $J=6)$. We choose the magnitude of the external field in (1) to be very large (5000 T) 
which constrains the magnetization to point along the chosen field direction $\hat{\mathbf{B}}$. The zero temperature (ground state) energy is thus obtained as a function of magnetization angle, which can be fitted to the expression 15 . In the case that the $l=2$ term dominates, the following relation is satisfied [42]:

$$
K_{1}=-3 J(J-0.5) \alpha_{J}\left[A_{20}\left\langle r^{2}\right\rangle\right] .
$$

For a general RE-TM magnet it is not straightforward to relate CF coefficients to the $K_{i}$ constants. This is because the CF coefficients only contain the contribution of the RE, but the TM will also contribute to the anisotropy [42]. However, making the reasonable assumption that the $\mathrm{RE}$ provides the dominant contribution, one can use the $\mathrm{CF}$ coefficients to at least make qualitative statements about the anisotropy. For instance, multiplying the signs of the Stevens factor $\alpha_{J}$ and $\left[A_{20}\left\langle r^{2}\right\rangle\right]$ gives the sign of negative $K_{1}$, assuming the $l=2$ term is dominant (16). Similarly, for cubic systems like the Laves phase compounds (where the first nonzero CF coefficient has $l=4)$, the product of $\left[A_{40}\left\langle r^{4}\right\rangle\right]$ and $\beta_{J}$ will be positive for an easy [111] direction and negative for [001]. The latter behaviour can be verified by plotting the potential $B_{40} Y_{40}(\theta)+B_{4 \pm 4} Y_{4 \pm 4}(\theta)$ in the $\phi=0$ plane multiplied by the sign of the Stevens factor, and seeing if the result is maximal or minimal along the [001] direction.

\subsection{Computational details}

We calculated the potential $V_{l m}^{\sigma}(r)$ including the PAW corrections (6) for the Yanalogues of RE-TM magnets using the GPAW code, version 1.4.0 [52]. We used the latest freely available GPAW PAW datasets (version 0.9.2) [56]. The calculations were performed using a plane wave basis set, expanding the wavefunctions up to a maximum plane wave kinetic energy of $1200 \mathrm{eV}$. The reciprocal space sampling was performed using $\Gamma$-centred $k$-point meshes and converged for each material, and the Kohn-Sham states occupied according to a Fermi-Dirac distribution with a width of $0.01 \mathrm{eV}$. The exchange-correlation energy was modelled with the LSDA [28]. The PAW corrections Appendix A were extracted using our own Python scripts.

To calculate the spherically-symmetric $4 f$ electron density $n_{4 f}^{0}(r)$ we used the Hutsepot KKR code 62] to calculate the spherically-symmetric potential at the RE site within the muffin-tin approximation. The muffin tin radii are reported in Table 1 . We used the LSDA + LSIC [36] to model the XC energy, where the angular momentum channels were corrected according to the scheme described in Ref. [31]. Using this potential we solved the atomic problem on a logarithmic radial grid to obtain the Green's function and density [(13) and (14)]. Finally, $V_{l m}^{\sigma}(r)$ and $n_{4 f}^{0}(r)$ were combined to calculate CF coefficients from (4) and converted into $\left[A_{l m}\left\langle r^{l}\right\rangle\right]$ notation [41]. 
Table 1. Structural parameters used in the calculations, with the Wyckoff positions occupied by each inequivalent atom given. Lattice parameters and muffin tin radii are in $\AA$, internal co-ordinates of the indicated Wyckoff positions are dimensionless.

\begin{tabular}{|c|c|c|c|c|}
\hline Material & $\begin{array}{l}\text { Space group; } \\
\text { RE site symmetry }\end{array}$ & $\begin{array}{l}\text { Lattice parameters; } \\
\text { MT radius }\end{array}$ & Internal co-ordinates & Source and reference \\
\hline $\mathrm{SmCo}_{5}$ & $\begin{array}{l}191(\mathrm{P} 6 / \mathrm{mmm}) \\
D_{6 h}\end{array}$ & $\begin{array}{l}a=4.974, c=3.978 \\
r_{\mathrm{MT}}=1.645\end{array}$ & $\operatorname{Sm}(1 a), \operatorname{Co}(2 c), \operatorname{Co}(3 g)$ & Exp. at $5 \mathrm{~K} 65$ \\
\hline $\mathrm{NdCo}_{5}$ & $\begin{array}{l}191(\mathrm{P} 6 / \mathrm{mmm}) \\
D_{6 h}\end{array}$ & $\begin{array}{l}a=5.006, c=3.978 \\
r_{\mathrm{MT}}=1.661\end{array}$ & $\mathrm{Nd}(1 a), \mathrm{Co}(2 c), \mathrm{Co}(3 g)$ & Exp. at $5 \mathrm{~K} 65$ \\
\hline $\mathrm{Sm}_{2} \mathrm{Co}_{17}$ & $\begin{array}{l}166(\mathrm{R} \overline{3} \mathrm{~m}) \\
C_{3 v}\end{array}$ & $\begin{array}{l}a=8.398, c=12.218 \\
r_{\mathrm{MT}}=1.781\end{array}$ & $\begin{array}{l}\operatorname{Sm}(6 c), \operatorname{Co}(6 c), \operatorname{Co}(9 d), \operatorname{Co}(18 f), \mathrm{Co}(18 h) \\
z_{6 c, \mathrm{Sm}}=0.343 ; z_{6 c, \mathrm{Co}}=0.099 ; x_{18 f}=0.283 \\
(x, z)_{18 h}=(0.171,0.486)\end{array}$ & $\begin{array}{l}\text { Exp. at } 300 \mathrm{~K}[66] \\
\left(\mathrm{Nd}_{2} \mathrm{Co}_{17} ; \text { see text }\right)\end{array}$ \\
\hline $\mathrm{NdFe}_{12}$ & $\begin{array}{l}139(\mathrm{I} 4 / \mathrm{mmm}) \\
D_{4 h}\end{array}$ & $\begin{array}{l}a=8.533, c=4.681 \\
r_{\mathrm{MT}}=1.667\end{array}$ & $\begin{array}{l}\mathrm{Nd}(2 a), \mathrm{Fe}(8 f), \mathrm{Fe}(8 i), \mathrm{Fe}(8 j) \\
x_{8 i}=0.359 ; x_{8 j}=0.268\end{array}$ & DFT-GGA 47. \\
\hline $\mathrm{NdFe}_{12} \mathrm{~N}$ & $\begin{array}{l}139(\mathrm{I} 4 / \mathrm{mmm}) \\
D_{4 h}\end{array}$ & $\begin{array}{l}a=8.521, c=4.883 \\
r_{\mathrm{MT}}=1.667\end{array}$ & $\begin{array}{l}\mathrm{Nd}(2 a), \mathrm{N}(2 b), \mathrm{Fe}(8 f), \mathrm{Fe}(8 i), \mathrm{Fe}(8 j) \\
x_{8 i}=0.361 ; x_{8 j}=0.274\end{array}$ & DFT-GGA 47] \\
\hline $\mathrm{SmFe}_{12}$ & $\begin{array}{l}139(\mathrm{I} 4 / \mathrm{mmm}) \\
D_{4 h}\end{array}$ & $\begin{array}{l}a=8.497, c=4.687 \\
r_{\mathrm{MT}}=1.667\end{array}$ & $\begin{array}{l}\mathrm{Sm}(2 a), \mathrm{Fe}(8 f), \mathrm{Fe}(8 i), \mathrm{Fe}(8 j) \\
x_{8 i}=0.359 ; x_{8 j}=0.270\end{array}$ & DFT-GGA 47 \\
\hline $\mathrm{SmFe}_{12} \mathrm{~N}$ & $\begin{array}{l}139(\mathrm{I} 4 / \mathrm{mmm}) \\
D_{4 h}\end{array}$ & $\begin{array}{l}a=8.517, c=4.844 \\
r_{\mathrm{MT}}=1.667\end{array}$ & $\begin{array}{l}\mathrm{Sm}(2 a), \mathrm{N}(2 b), \mathrm{Fe}(8 f), \mathrm{Fe}(8 i), \mathrm{Fe}(8 j) \\
x_{8 i}=0.361 ; x_{8 j}=0.274\end{array}$ & DFT-GGA 47 \\
\hline $\mathrm{TbFe}_{2}$ & $227(\mathrm{Fd} \overline{3} \mathrm{~m}) ; T_{d}$ & $a=7.341, r_{\mathrm{MT}}=1.589$ & $\mathrm{~Tb}(8 b), \mathrm{Fe}(16 c)$ & Exp. at $300 \mathrm{~K} 65$ \\
\hline $\mathrm{DyFe}_{2}$ & $227(\mathrm{Fd} \overline{3} \mathrm{~m}) ; T_{d}$ & $a=7.338, r_{\mathrm{MT}}=1.589$ & $\mathrm{Dy}(8 b), \mathrm{Fe}(16 c)$ & Exp. at $300 \mathrm{~K} 65$ \\
\hline $\mathrm{Tb}$ & $\begin{array}{l}194\left(\mathrm{P} 6_{3} / \mathrm{mmc}\right) \\
D_{3 h}\end{array}$ & $\begin{array}{l}a=3.606, c=5.698 \\
r_{\mathrm{MT}}=1.764\end{array}$ & $\operatorname{Tb}(2 c)$ & Exp. at $300 \mathrm{~K} 67$ \\
\hline
\end{tabular}

\section{Results}

\subsection{Materials considered}

In Table 1 we list the 10 materials considered in this work. In our set of materials we have included the archetypal Sm-Co magnets $\mathrm{SmCo}_{5}$ [7] and $\mathrm{Sm}_{2} \mathrm{Co}_{17}$ [8], and also examples of the 1:12 magnet class e.g. $\mathrm{NdFe}_{12} \mathrm{~N}$, which are currently the subject of research due to their potential as hard magnetic materials with reduced RE content [68]. We have also included the Laves phase magnets $\mathrm{TbFe}_{2}$ and $\mathrm{DyFe}_{2}$ whose alloy is the highly magnetostrictive Terfenol-D compound [69, 70], and also elemental $\mathrm{Tb}$ [71]. Tb is an interesting test since the Y-analogue has no atoms in common with the original structure.

Table 1 also gives the structural parameters (lattice constants and internal coordinates) used in the calculations. In order to be able to best compare our CF coefficients to previous calculations we have where possible used the same structural parameters. Accordingly, as indicated in Table 1 structural parameters have been sourced both from experimental and computational works. For $\mathrm{Sm}_{2} \mathrm{Co}_{17}$ we follow Ref. [46] and use the structural parameters of the related compound $\mathrm{Nd}_{2} \mathrm{Co}_{17}$ [66], which avoids the experimental difficulties associated with performing neutron experiments on Sm compounds.

As clearly explained in Ref. [42, depending on the point symmetry of the RE site only certain CF coefficients will be nonzero. Furthermore, for the purposes of calculating matrix elements for $f$ electrons we need only calculate CF coefficients for even values of $l$, up to a maximum $l=6$. In order to check that our computational method is robust we 
Table 2. Calculated crystal field coefficients, in K (i.e. $\left[A_{l m}\left\langle r^{l}\right\rangle\right] / k_{B}$ ). The two numbers reported for each $l m$ combination corresponds to spin up/down respectively, and the bold number corresponds to the spin of the asymmetric $4 f$ electron cloud, as discussed in the text.

\begin{tabular}{|c|c|c|c|c|c|c|c|c|}
\hline Material & {$\left[A_{20}\left\langle r^{2}\right\rangle\right]$} & {$\left[A_{40}\left\langle r^{4}\right\rangle\right]$} & {$\left[A_{43}\left\langle r^{4}\right\rangle\right]$} & {$\left[A_{44}\left\langle r^{4}\right\rangle\right]$} & {$\left[A_{60}\left\langle r^{6}\right\rangle\right]$} & {$\left[A_{63}\left\langle r^{6}\right\rangle\right]$} & {$\left[A_{64}\left\langle r^{6}\right\rangle\right]$} & {$\left[A_{66}\left\langle r^{6}\right\rangle\right]$} \\
\hline $\mathrm{SmCo}_{5}$ & $-402 /-400$ & $-30 /-22$ & - & - & $5 / 4$ & - & - & $-137 /-\mathbf{1 1 5}$ \\
\hline DMFT 20] & $-313 /-\mathbf{2 6 2}$ & $-40 /-55$ & - & - & $35 / 25$ & - & - & $-731 /-593$ \\
\hline $\mathrm{NdCo}_{5}$ & $-421 /-415$ & $-36 /-26$ & - & - & $6 / 5$ & 一 & - & $-174 /-\mathbf{1 4 6}$ \\
\hline $\mathrm{Sm}_{2} \mathrm{Co}_{17}$ & $-199 /-208$ & $-20 /-7$ & $116 / \mathbf{1 0 8}$ & - & $-1 /-1$ & $-22 /-\mathbf{- 2 5}$ & 一 & $-54 /-48$ \\
\hline open core 46 & -194 & -15 & 74 & 一 & -2 & -61 & - & -139 \\
\hline $\mathrm{NdFe}_{12}$ & $-116 /-110$ & $4 / \mathbf{1 5}$ & - & $-205 /-145$ & $10 / 7$ & - & $-29 /-21$ & - \\
\hline open core 47. & -77 & - & - & - & - & - & - & - \\
\hline DMFT [20] & $-71 /-116$ & $-5 /-1$ & - & $-76 /-270$ & $62 / \mathbf{5 4}$ & - & $-224 /-107$ & - \\
\hline $\mathrm{NdFe}_{12} \mathrm{~N}$ & $188 / \mathbf{3 6 4}$ & $-62 /-\mathbf{1 3}$ & - & $-161 /-\mathbf{1 0 1}$ & $-17 /-16$ & - & $-1 /-\mathbf{3}$ & - \\
\hline open core 47 & 367 & - & - & - & - & - & - & - \\
\hline DMFT [20] & $477 / \mathbf{6 5 3}$ & $75 / 112$ & - & $-105 /-141$ & $32 / \mathbf{6 3}$ & - & $-65 /-91$ & - \\
\hline $\mathrm{SmFe}_{12}$ & $-100 /-96$ & $1 / \mathbf{1 0}$ & - & $-154 /-112$ & $8 / 6$ & - & $-21 /-15$ & - \\
\hline open core 47 & -47 & - & - & - & - & - & - & - \\
\hline DMFT [20] & $-184 /-211$ & $-21 / \mathbf{- 1 8}$ & - & $-41 /-\mathbf{1 3 6}$ & $45 / \mathbf{4 0}$ & - & $-95 /-58$ & - \\
\hline $\mathrm{SmFe}_{12} \mathrm{~N}$ & $272 / \mathbf{4 1 4}$ & $-47 /-6$ & - & $-121 /-\mathbf{7 5}$ & $-14 /-\mathbf{1 3}$ & - & $-2 /-\mathbf{3}$ & - \\
\hline open core 47 & 371 & - & - & - & - & - & - & - \\
\hline DMFT [20] & $195 / \mathbf{2 2 5}$ & $78 / \mathbf{7 0}$ & - & $22 /-\mathbf{9 1}$ & $47 / \mathbf{2 5}$ & - & $-97 /-82$ & - \\
\hline $\mathrm{TbFe}_{2}$ & - & $\mathbf{2 8} / 29$ & - & 139/143 & $-2 /-2$ & - & $\mathbf{3 9} / 38$ & - \\
\hline $\mathrm{DyFe}_{2}$ & - & $\mathbf{2 6} / 26$ & - & $\mathbf{1 2 8} / 132$ & $-2 /-2$ & - & $\mathbf{3 4} / 32$ & - \\
\hline $\mathrm{Tb}$ & $-59 /-\mathbf{6 0}$ & $-4 /-3$ & - & - & $4 / 4$ & - & - & $36 / \mathbf{3 6}$ \\
\hline
\end{tabular}

calculated the decomposition of the potential (6) for all $\mathrm{lm}$ combinations and confirmed that the only nonzero terms were those expected from the point symmetry [61].

\subsection{CF coefficients}

Table 2 gives the CF coefficients calculated for our set of materials. We also reproduce the values of $\mathrm{CF}$ coefficients calculated previously in the literature for some of the materials [20, 46, 47].

For our calculations, and Ref. [20], two numbers are reported for each $\mathrm{lm}$, corresponding to the CF coefficient calculated using $V_{l m}^{\uparrow}(r)$ and $V_{l m}^{\downarrow}(r)$. One of the two numbers has been written in bold, corresponding to the coefficient that (in the zero temperature, scalar-relativistic picture) is the potential felt by the partially filled If spin subshell. Expanding on this point, we first note that we have chosen the TM spins to point in the $\uparrow$ direction. Furthermore, the RE-TM coupling is generally antiferromagnetic between spins [5]. Therefore, for the light $\mathrm{REs} \mathrm{Nd}$ and $\mathrm{Sm}$ the partially filled $4 f$ subshell has $\downarrow$ spin character, so the relevant $\mathrm{CF}$ coefficient is calculated using $V_{l m}^{\downarrow}(r)$. For the heavy REs, the $4 f \downarrow$ spin subshell is filled, so $V_{l m}^{\uparrow}(r)$ is relevant for $\mathrm{TbFe}_{2}$ and $\mathrm{DyFe}_{2}$. For elemental $\mathrm{Tb}$ we chose $\uparrow$ to be the majority spin direction, so the unfilled $4 f$ spin subshell is $\downarrow$. We note that, except for $\mathrm{NdFe}_{12} \mathrm{~N}$ and $\mathrm{SmFe}_{12} \mathrm{~N}$, we calculate the difference between $V_{l m}^{\uparrow}(r)$ and $V_{l m}^{\downarrow}(r)$ to be rather small. We now discuss each material in more detail. 
3.2.1. $\mathrm{SmCO}_{5}$ and $\mathrm{NdCO}_{5} \mathrm{SmCo}_{5}$ crystallizes in the $\mathrm{CaCu}_{5}$ structure, which is hexagonal with one formula unit in the unit cell [9]. $\mathrm{SmCo}_{5}$ is characterized by a large uniaxial anisotropy [72, which is understood in terms of CF theory based on the approximate relation $(16)$ between the first anisotropy constant and lowest order CF coefficient, $K_{1} \propto-\alpha_{J}\left[A_{20}\left\langle r^{2}\right\rangle\right]$ [42]. The positive value of $13 / 315$ for the Stevens $\alpha_{J}$ coefficient of $\mathrm{Sm}^{3+}$ [42] means that $\mathrm{SmCo}_{5}$ should have a large, negative value of $\left[A_{20}\left\langle r^{2}\right\rangle\right][73]$.

Recently, Ref. [20] calculated the CF coefficients of $\mathrm{SmCo}_{5}$ within DMFT, and also provided a useful summary of past calculations and experimental measurements. Experimentally measured values of $\left[A_{20}\left\langle r^{2}\right\rangle\right]$ vary between -180 and $-420 \mathrm{~K}$, whilst calculations found values between -160 and $-755 \mathrm{~K}$. There is no particular consensus about the other CF coefficients, although the computational works agreed in finding $\left[A_{40}\left\langle r^{4}\right\rangle\right]$ to be negative and $\left[A_{60}\left\langle r^{6}\right\rangle\right]$ to be positive. We reproduce the results of the DMFT calculations with our own results in Table 2 .

Our calculations based on the Y-analogue model of $\mathrm{SmCo}_{5}$ find values of the $\left[A_{20}\left\langle r^{2}\right\rangle\right]$ coefficient $(-402 /-400 \mathrm{~K})$ which fall into the previously reported experimental/calculated ranges. The variation in sign of $\left[A_{20}\left\langle r^{2}\right\rangle\right],\left[A_{40}\left\langle r^{4}\right\rangle\right]$ and $\left[A_{60}\left\langle r^{6}\right\rangle\right]$ also follows that observed in previous calculations. Despite the different methodologies involved, our calculated values are reasonably close to the DMFT results [20], with the exception of $\left[A_{66}\left\langle r^{6}\right\rangle\right]$. This CF coefficient controls the basal plane anisotropy. However, it does not affect the ground- $J$ multiplet of Sm [42]. Furthermore, higher order $\mathrm{CF}$ coefficients decay more quickly with temperature [42]. Therefore, the effect of $\left[A_{66}\left\langle r^{6}\right\rangle\right]$ on the strongly uniaxial $\mathrm{SmCo}_{5}$ is expected to be difficult to observe, and has not been measured experimentally [73].

For comparison we also considered the isostructural $\mathrm{NdCO}_{5}$ compound. Within the Y-analogue model, any differences between $\mathrm{CF}$ coefficients calculated for $\mathrm{SmCo}_{5}$ and $\mathrm{NdCo}_{5}$ must be attributed to (a) the slightly different lattice parameters (Table 1) and (b) the different $4 f$ electron density (Fig. 1). We see from Table 2 that the calculated CF coefficients are actually very similar for both materials. However, $\mathrm{Nd}^{3+}$ has a negative Stevens factor $(-7 / 1089)$ [42], which means $\mathrm{NdCo}_{5}$ should have an in-plane anisotropy (negative $K_{1}$ ), This is exactly what is observed experimentally [74]. Furthermore, unlike $\mathrm{SmCo}_{5}$ the anisotropy within the basal plane has also been measured experimentally, with the easy direction found to point between Co atoms [64] $\left[\phi=30^{\circ}\right.$ in Fig. 2(a)]. This easy direction is indeed consistent with the negative $\left[A_{66}\left\langle r^{6}\right\rangle\right]$ which we calculate, since the Stevens $\gamma_{J}(l=6)$ coefficient of $\mathrm{Nd}^{3+}$ is negative [42].

3.2.2. $\mathrm{Sm}_{2} \mathrm{Co}_{17}$ The magnet $\mathrm{Sm}_{2} \mathrm{Co}_{17}$ forms in the $\mathrm{Th}_{2} \mathrm{Zn}_{17}$ structure. This structure is closely related to $\mathrm{SmCo}_{5}$ by an ordered substitution of one in three $\mathrm{Sm}$ atoms with a pair (dumbbell) of Co atoms [9]. Although there is a distortion of the local environment, the Sm atoms are still surrounded by a hexagon of effectively coplanar Co atoms, as shown in Fig. 2(b). However, as is also clear from Fig. 2(b) the dumbbells lower the hexagonal symmetry so that $\mathrm{CF}$ coefficients with $m= \pm 3$ are nonzero. What is not 
shown in Fig. 2 is the tripling of the unit cell along the $c$ direction compared to $\mathrm{SmCo}_{5}$, due to the stacking of two Sm atoms and a dumbbell. Again we stress that our choice of axes in Fig. 2(b) is unconventional compared to Fig. 2(a), but physically gives a closer correspondence between the $(\theta, \phi)$ co-ordinate system and the positions of atoms in $\mathrm{SmCo}_{5}$.

Now considering the calculated $\left[A_{l m}\left\langle r^{l}\right\rangle\right]$ in Table 2 , we see that introducing the Co dumbbells results in reduced coefficients compared to $\mathrm{SmCo}_{5}$. In particular, $\left[A_{20}\left\langle r^{2}\right\rangle\right]$ is halved. The value of $-208 \mathrm{~K}$ is still negative however, supporting uniaxial anisotropy as is observed experimentally [9]. In terms of anisotropy within the basal plane, $\left[A_{66}\left\langle r^{6}\right\rangle\right]$ is negative like in $\mathrm{SmCO}_{5}$, but is weaker by a factor of 2 .

Ref. [46] reported calculations of the $\mathrm{CF}$ coefficients of $\mathrm{Sm}_{2} \mathrm{Co}_{17}$, modelling the $\mathrm{Sm}$ in the $3+$ state with the open-core approximation. We reproduce the calculated values in Table 2, and find very close agreement with our Y-analogue model. The exception is for the $l=6$ coefficients, with Ref. 46] finding values a factor of 3 larger than our calculations. As for $\mathrm{SmCo}_{5}$, there is no experimental data for these coefficients. However, we think that it is reasonable that our calculations show a reduction in $\left[A_{66}\left\langle r^{6}\right\rangle\right]$ for $\mathrm{Sm}_{2} \mathrm{Co}_{17}$ compared to $\mathrm{SmCo}_{5}$, due to the disrupted hexagonal symmetry.

3.2.3. $\mathrm{NdFe}_{12}, \mathrm{NdFe}_{12} \mathrm{~N}, \mathrm{SmFe}_{12}, \mathrm{SmFe}_{12} \mathrm{~N}$ Members of the 1:12 magnet class have a tetragonal, $\mathrm{ThMn}_{12}$ crystal structure with the RE surrounded by $3 \mathrm{TM}$ sublattices. The interstitial $2 b$ positions can also be occupied by nonmetal atoms, forming e.g. $\mathrm{NdFe}_{12} \mathrm{~N}[68$. The fourfold symmetry in the $a b$ plane gives rise to nonzero CF coefficients with $m \pm 4$. However, it is the lowest order coefficient $\left[A_{20}\left\langle r^{2}\right\rangle\right]$ (which decays the slowest with temperature) which is expected to have the largest effect on the uniaxial anisotropy. As for the materials above, a negative value of $\left[A_{20}\left\langle r^{2}\right\rangle\right]$ is expected to yield uniaxial anisotropy for Sm and planar anisotropy for $\mathrm{Nd}$, based on the sign of the Stevens coefficient.

Table 2 shows our calculated CF coefficients for $\mathrm{NdFe}_{12}, \mathrm{NdFe}_{12} \mathrm{~N}, \mathrm{SmFe}_{12}$ and $\mathrm{SmFe}_{12} \mathrm{~N}$. The most striking feature is the sign reversal of $\left[A_{20}\left\langle r^{2}\right\rangle\right]$ upon nitrogenation, switching from negative to positive. These signs mean that $\mathrm{SmFe}_{12}$ and $\mathrm{NdFe}_{12} \mathrm{~N}$ should have uniaxial anisotropy, and $\mathrm{NdFe}_{12}$ and $\mathrm{SmFe}_{12} \mathrm{~N}$ should be planar. Nitrogenation also provides a negative contribution to $\left[A_{40}\left\langle r^{4}\right\rangle\right]$, but weakens $\left[A_{44}\left\langle r^{4}\right\rangle\right]$. The $l=6 \mathrm{CF}$ coefficents are small for all of the materials.

Also in Table 2 we reproduce reported CF coefficients calculated with DMFT [20] and with the open core approximation [47] (only the $l=2$ coefficients are reported in Ref. [47]). Focusing first on $\left[A_{20}\left\langle r^{2}\right\rangle\right]$ we find that the three methods calculate the same signs. Furthermore there is close numerical agreement between our Y-analogue calculations and the open core calculations [47], as for $\mathrm{Sm}_{2} \mathrm{Co}_{17}$. However, there is no systematic level of agreement of these calculations with DMFT, with for instance similar values calculated for $\mathrm{NdFe}_{12}$ but variations up to a factor of two for the other materials. The agreement between the Y-analogue model and DMFT for the higher order CF coefficients is similarly unsystematic, with for instance opposite signs found for $\left[A_{40}\left\langle r^{4}\right\rangle\right]$ 
but quite close agreement for $\left[A_{44}\left\langle r^{4}\right\rangle\right]$. Like for $\mathrm{SmCo}_{5}$, the DMFT calculations find larger values of $l=6$ coefficients than in the Y-analogue model.

We note that both the DMFT and Y-analogue calculations find that nitrogenation introduces a significant difference between the $\uparrow$ and $\downarrow \mathrm{CF}$ coefficients. At first sight, this observation is puzzling since $\mathrm{N}$ is nonmagnetic. However, as shown in Ref. [47] the introduction of $\mathrm{N}$ strengthens the magnetization by approximately $2.5 \mu_{\mathrm{B}}$ per formula unit, which our calculations find is due to an enhanced Fe magnetization at the $8 f$ sites. The $8 f$ sites sit halfway between RE planes, so it is not unreasonable that an enhanced spin polarization here will affect the $\uparrow$ and $\downarrow$ CF coefficients by differing amounts.

Finally, comparing the $\mathrm{Y}$-analogue calculations between $\mathrm{Nd}$ and $\mathrm{Sm}$ compounds we see that the same qualitative behavior of the CF coefficients is observed. However, unlike for $\mathrm{SmCo}_{5}$ and $\mathrm{NdCo}_{5}$ there are some numerical differences, particularly for the nitrided compounds where there is a large difference in $c$ parameter for $\mathrm{NdFe}_{12} \mathrm{~N}$ and $\mathrm{SmFe}_{12} \mathrm{~N}$.

3.2.4. $\mathrm{TbFe}_{2}$ and $\mathrm{DyFe}_{2}$ The Laves phase $\mathrm{REFe}_{2}$ compounds are notable for their large room temperature magnetostrictions, particularly the alloy $\mathrm{Tb}_{0.27} \mathrm{Dy}_{0.73} \mathrm{Fe}_{2}$ (TerfenolD) [69, 70]. Unlike the other materials in our test set the Laves phase $\left(\mathrm{MgCu}_{2}\right)$ structure is cubic, yielding a zero $\left[A_{20}\left\langle r^{2}\right\rangle\right]$ coefficient. They are also key examples of RE-TM magnets based on heavy REs with practical applications.

In Table 2 we give the CF coefficients calculated using the Y-analogue model for $\mathrm{TbFe}_{2}$ and $\mathrm{DyFe}_{2}$. We point out that although four coefficients are presented for each material, only $\left[A_{40}\left\langle r^{4}\right\rangle\right]$ and $\left[A_{60}\left\langle r^{6}\right\rangle\right]$ are independent, with the cubic symmetry fixing the ratios $B_{44}: B_{40}$ and $B_{64}: B_{60}$ [61]. The first notable feature is that the calculated coefficients are almost identical for $\mathrm{TbFe}_{2}$ and $\mathrm{DyFe}_{2}$. As a consequence, within CF theory any different behavior of the anisotropy must come from the Stevens factors.

The $\beta_{J}(l=4)$ factors are 2/16335 and -8/135135 for $\mathrm{Tb}^{3+}$ and $\mathrm{Dy}^{3+}$, respectively. The $\gamma_{J}$ coefficients also have opposite signs [42]. Therefore, one would expect $\mathrm{TbFe}_{2}$ and $\mathrm{DyFe}_{2}$ to have different easy directions of magnetization, either [111] or [100]. Specifically, based on the positive $l=4 \mathrm{CF}$ coefficients one would expect an easy direction of [111] for $\mathrm{TbFe}_{2}$ and [100] for $\mathrm{DyFe}_{2}$ (Sec. 2.9). This behaviour is exactly what is observed experimentally; indeed the easy directions of all of the heavy $\mathrm{RFe}_{2}$ compounds follow the sign of $\beta_{J}$ [65].

To our knowledge the CF coefficients of $\mathrm{TbFe}_{2}$ and $\mathrm{DyFe}_{2}$ have not been calculated from first principles previously. Experimental determination of these cubic terms is also expected to be difficult due to the large magnetostrictive effect. For example, based on Mössbauer measurements the authors of Ref. [75] were unable to determine precise values of $\left[A_{l m}\left\langle r^{l}\right\rangle\right]$, except that $A_{40}$ was of order $10 \mathrm{~K} / a_{0}^{4}$. Furthermore, the ratio $A_{60} / A_{40}$ was determined to be $-0.04 a_{0}^{-2}$. As pointed out in Sec. 2.8 , we cannot extract $A_{l m}$ from $\left\langle r^{l}\right\rangle$ so a direct comparison is not possible. However, our value of $\left[A_{40}\left\langle r^{4}\right\rangle\right]$ is also of order $10 \mathrm{~K}$, and our calculated ratio $\left[A_{60}\left\langle r^{6}\right\rangle\right] /\left[A_{40}\left\langle r^{4}\right\rangle\right]$ is -0.08 . Therefore we can at least say that the sign of the ratio of the $l=4$ and $6 \mathrm{CF}$ coefficients is consistent with 
Ref. [75], and also that the magnitudes are reasonable.

3.2.5. $\mathrm{Tb}$ The final material we consider is elemental Tb. Here, every atom in the system is replaced with $\mathrm{Y}$, so arguably this material presents the most difficult challenge

to the $\mathrm{Y}$-analogue model, even though the nominal valence electron configurations are the same.

$\mathrm{Tb}$ is hexagonal, and has the same nonzero $\mathrm{CF}$ coefficients as $\mathrm{NdCo}_{5}$. Indeed, as seen in Table 2 the relative magnitudes of the different $\mathrm{CF}$ coefficients of $\mathrm{Tb}$ follow those of $\mathrm{NdCO}_{5}$ reasonably closely, except for the crucial difference of $\left[A_{66}\left\langle r^{6}\right\rangle\right]$, which is positive for $\mathrm{Tb}$. Noting that $\mathrm{Tb}^{3+}$ has negative Stevens $\alpha_{J}$ and $\gamma_{J}$ coefficients like $\mathrm{Nd}^{3+}$, the calculated CF coefficients lead to easy plane anisotropy, with a preferred direction in the basal plane of $\phi=0$, i.e. pointing between the nearest in-plane Tb atoms [Fig. 2(a)]. The calculated easy direction matches that observed experimentally [64].

Unlike the other materials in our test set, there are no other magnetic sublattices present in $\mathrm{Tb}$. Therefore, we can use the method described in Sec. 2.9 to calculate anisotropy constants at $0 \mathrm{~K}$, obtaining values of $-17,-12$ and $5 \mathrm{MJm}^{-3}$ for $K_{1}, K_{2}$ and $K_{3}$, and $-0.2 \mathrm{MJm}^{-3}$ for $K_{4}$. These numbers are smaller than the values reported from low temperature high-field measurements, e.g. $-60 \mathrm{MJm}^{-3}$ for $K_{1}$ only, and $-2.4 \mathrm{MJ}$ for $K_{4}$ [71, 76]. However it is important to remember that our calculations do not include magnetostrictive effects, which are highly important in Tb. For instance, the high-field magnetization curve clearly shows a hysteresis, which has been attributed to a plastic deformation of the crystal [76].

\section{Conclusions and outlook}

Aside from the numerical results of our work contained in Table 2, we can summarize our results qualitatively as follows:

- The signs of our calculated CF coefficients are consistent with experimentallyobserved/previously calculated magnetization directions for all 10 materials (easy axis: $\mathrm{SmCo}_{5}, \mathrm{Sm}_{2} \mathrm{Co}_{17}, \mathrm{NdFe}_{12} \mathrm{~N}, \mathrm{SmFe}_{12}$; easy plane: $\mathrm{NdCo}_{5}, \mathrm{NdFe}_{12}, \mathrm{SmFe}_{12} \mathrm{~N}$, $\mathrm{Tb} ;[111]$ axis: $\mathrm{TbFe}_{2} ;[001]$ axis, $\mathrm{DyFe}_{2}$ ).

- For systems with data available $\left(\mathrm{NdCo}_{5}\right.$ and $\left.\mathrm{Tb}\right)$, the signs of the $\left[A_{66}\left\langle r^{6}\right\rangle\right]$ coefficients are also consistent with the experimentally-observed basal plane anisotropy.

- Our calculated CF coefficients are generally close (within $50 \mathrm{~K} / 4 \mathrm{meV}$ ) of previously reported open core calculations.

- The trend across the Sm-Co series behaves intuitively, with CF coefficients weakened for lower symmetry $\left(\mathrm{SmCo}_{5} \mathrm{vs}_{2} \mathrm{Co}_{17}\right)$.

Taken together, our results demonstrate that the Y-analogue model is a viable method of calculating CF coefficients of RE-TM magnets. Of course, it is not the only method, and we do not claim that it is any more accurate than previously published 
work [12, 17, 20, 44, 45, 46, 47, 48]. In our opinion the strength of the Y-analogue model is in the simplicity of the calculation of the CF potential $V(\mathbf{r})$. Whilst implemented in some DFT codes like OpenMX [77], the open-core approximation (which appears to give similar CF coefficients to the Y-analogue model), is not yet universally available. By contrast, the PAW dataset for $\mathrm{Y}$ is distributed as standard in all of the popular DFT codes. We also find the calculations involving $\mathrm{Y}$ to be numerically stable. As such, we would expect a high degree of reproducibility of the CF potential calculated for Yanalogues using different codes, similar to that demonstrated e.g. for bulk moduli [78].

As noted in Sec. 2, the CF potential must be supplemented by the radial $4 f$ charge density $n_{4 f}^{0}(r)$ in order to calculate the CF coefficient. Unfortunately, calculating $n_{4 f}^{0}(r)$ is not completely straightforward, requiring a way of dealing with the $4 f$ electrons like the LSIC. However, as we argued in the discussion surrounding Fig. 1, the same $n_{4 f}^{0}(r)$ could be used to calculate the CF coefficients for a range of compounds containing a given lanthanide. This further approximation would then mean only the CF potential needed to be calculated for each compound, enabling high throughput computational screening of materials based on their calculated CF coefficients and scaling up the work of e.g. Refs. [19, 26].

From the point of view of assessing the predictive power of our calculations, the most interesting avenue for future exploration is to go beyond calculating $\mathrm{CF}$ coefficients and instead target quantities that can be directly compared to experiments, e.g. magnetization versus field curves, from which anisotropy constants $K_{i}$ (15) are extracted. Although we tentatively discussed anisotropy constants for $\mathrm{Tb}$, our ability to compare to experiment was limited due to us not taking any magnetoelastic effects into account. Including such effects will be crucial if we are to properly account for the magnetostriction of the heavy RE materials, including elemental $\mathrm{Tb}$ [71, 76] and the Laves phase $\mathrm{Tb}_{1-x} \mathrm{Dy}_{x} \mathrm{Fe}_{2}$ compounds [69].

More generally, we did not attempt to determine $K_{i}$ for the RE-TM compounds here since the $\mathrm{CF}$ coefficients do not include the contribution to the anisotropy from the TM. However, we have previously calculated the TM contribution for $\mathrm{GdCo}_{5}$ (which has no crystal field effects) and obtained values of $K_{i}$ in quantitative agreement with experiment, over a range of temperatures [79]. Therefore, by combining the method

presented here with our previous work [79] and finite temperature CF theory [42], we should have the necessary tools to calculate temperature-dependent anisotropy constants of RE-TM magnets entirely from first principles.

\section{Acknowledgments}

The present work forms part of the PRETAMAG project, funded by the UK Engineering and Physical Sciences Research Council, Grant No. EP/M028941/1. We thank J. J. Mortensen for guidance with the GPAW code. 


\section{Appendix A. Explicit expression for correction to the pseudo-density}

Here we give the explicit expression for the angular expansion of the correction to the pseudo-density. We use standard GPAW notation [52]. The difference between the full and pseudized density $\Delta \rho(\mathbf{r})$ is the sum of the electronic contribution $\Delta n(\mathbf{r})$ and a nuclear part:

$$
\Delta \rho(\mathbf{r})=\Delta n(\mathbf{r})-Z \delta(\mathbf{r})-\sum_{l m} Q_{l m} g_{l m}(\mathbf{r})
$$

Here, $Z$ is the nuclear charge (a positive number), $Q_{l m}$ are the compensation charges used to ensure the multipole moments of $\Delta \rho(\mathbf{r})$ are zero, and $g_{l m}$ are functions which in GPAW are Gaussians multiplied by real spherical harmonics. The spin-resolved correction for the electronic density is

$$
\begin{aligned}
\Delta n^{\sigma}(\mathbf{r})= & \frac{n_{c}(r)-\tilde{n}_{c}(r)}{2}+ \\
& \sum_{i_{1} i_{2}} D_{\sigma i_{1} i_{2}}\left[\phi_{i_{1}}(\mathbf{r}) \phi_{i_{2}}(\mathbf{r})-\tilde{\phi}_{i_{1}}(\mathbf{r}) \tilde{\phi}_{i_{2}}(\mathbf{r})\right] .
\end{aligned}
$$

Here, $n_{c}$ and $\tilde{n}_{c}$ are the full and pseudo core densities. $D$ is the atomic density matrix and

$\phi, \tilde{\phi}$ are the partial waves, which together allow the reconstruction of the wavefunction in the rapidly-varying region close to the nucleus. $i$ is a composite index standing for $(\nu, l, m)$ where $\nu$ plays the role of the principal quantum number of the partial waves.

$Z, g_{l m}, n_{c}, \tilde{n}_{c}, \phi$ and $\tilde{\phi}$ are properties of the PAW dataset, while $Q_{l m}$ and $D_{\sigma i_{1} i_{2}}$ are determined in the self-consistent calculation. Noting that the compensation charges and the partial waves have the forms $g_{l m}(\mathbf{r})=g_{l}(r) Y_{l m}^{R}(\hat{\mathbf{r}})$ and $\phi_{i_{1}}(\mathbf{r})=\phi_{\nu l}(r) Y_{l m}^{R}(\hat{\mathbf{r}})$ respectively, with the $R$ denoting real spherical harmonics, the angular expansions of $\Delta \rho$ and $\Delta n^{\sigma}$ are readily obtained. The only slight complication is the use of real spherical harmonics in GPAW, which means that one must take some care with integrals like $\int d \hat{\mathbf{r}} Y_{l m}^{*}(\hat{\mathbf{r}}) Y_{l^{\prime} m^{\prime}}^{R}(\hat{\mathbf{r}})$ and $\int d \hat{\mathbf{r}} Y_{l m}^{*}(\hat{\mathbf{r}}) Y_{l_{1} m_{1}}^{R}(\hat{\mathbf{r}}) Y_{l_{2} m_{2}}^{R}(\hat{\mathbf{r}})$.

\section{References}

[1] Hirosawa S 2018 Scr. Mater. 154245

[2] Gutfleisch O, Willard M A, Brück E, Chen C H, Sankar S G and Liu J P 2011 Adv. Mater. 23821

[3] Coey J M D 2011 IEEE Trans. Magn. 474671

[4] Gignoux D and Schmitt D 1995 Chapter 138 magnetic properties of intermetallic compounds (Handbook on the Physics and Chemistry of Rare Earths vol 20) pp 293 - 424

[5] Brooks M S S, Eriksson O and Johansson B 1989 J. Phys. Condens. Matter 15861

[6] Buschow K H J 1977 Rep. Prog. Phys. 401179

[7] Strnat K, Hoffer G, Olson J, Ostertag W and Becker J J 1967 J. Appl. Phys. 381001

[8] Strnat K 1972 IEEE Trans. Magn. 8511

[9] Kumar K 1988 J. Appl. Phys. 63 R13

[10] Sagawa M, Fujimura S, Togawa N, Yamamoto H and Matsuura Y 1984 J. Appl. Phys. 552083

[11] Croat J J, Herbst J F, Lee R W and Pinkerton F E 1984 J. Appl. Phys. 552078

[12] Richter M 1998 J. Phys. D: Appl. Phys. 311017

[13] Larson P, Mazin I I and Papaconstantopoulos D A 2003 Phys. Rev. B 67214405

[14] Kashyap A, Skomski R, Sabiryanov R F, Jaswal S S and Sellmyer D J 2003 IEEE Trans. Magn. 392908 
[15] Miura Y, Tsuchiura H and Yoshioka T 2014 J. Appl. Phys. 115 17A765

[16] Matsumoto M, Banerjee R and Staunton J B 2014 Phys. Rev. B 90054421

[17] Miyake T, Terakura K, Harashima Y, Kino H and Ishibashi S 2014 J. Phys. Soc. Jpn. 83043702

[18] Harashima Y, Terakura K, Kino H, Ishibashi S and Miyake T 2015 Phys. Rev. B 92184426

[19] Körner W, Krugel G and Elssser C 2016 Sci. Rep. 624686

[20] Delange P, Biermann S, Miyake T and Pourovskii L 2017 Phys. Rev. B 96155132

[21] Chouhan R K and Paudyal D 2017 J. Alloys Compd 723208

[22] Patrick C E, Kumar S, Balakrishnan G, Edwards R S, Lees M R, Mendive-Tapia E, Petit L and Staunton J B 2017 Phys. Rev. Mater. 1024411

[23] Toga Y, Nishino M, Miyashita S, Miyake T and Sakuma A 2018 Phys. Rev. B 98054418

[24] Sakurai M, Wu S, Zhao X, Nguyen M C, Wang C Z, Ho K M and Chelikowsky J R 2018 Phys. Rev. Mater. 2084410

[25] Miyake T and Akai H 2018 J. Phys. Soc. Jpn. 87041009

[26] Tatetsu Y, Harashima Y, Miyake T and Gohda Y 2018 Phys. Rev. Mater. 2074410

[27] Kohn W and Sham L J 1965 Phys. Rev. 140 A1133

[28] Vosko S H, Wilk L and Nusair M 1980 Can. J. Phys. 581200

[29] Perdew J P, Burke K and Ernzerhof M 1996 Phys. Rev. Lett. 773865

[30] Richter M and Eschrig H 1991 Physica B: Condens. Matter 17285

[31] Patrick C E and Staunton J B 2018 Phys. Rev. B 97224415

[32] Brooks M S S, Nordstrom L and Johansson B 1991 J. Phys. Condens. Matter 32357

[33] Kotliar G, Savrasov S Y, Haule K, Oudovenko V S, Parcollet O and Marianetti C A 2006 Rev. Mod. Phys. $\mathbf{7 8} 865$

[34] Perdew J P and Zunger A 1981 Phys. Rev. B 235048

[35] Anisimov V I, Aryasetiawan F and Lichtenstein A I 1997 J. Phys. Condens. Matter 9767

[36] Lüders M, Ernst A, Däne M, Szotek Z, Svane A, Ködderitzsch D, Hergert W, Györffy B L and Temmerman W M 2005 Phys. Rev. B 71205109

[37] Györffy B L, Pindor A J, Staunton J, Stocks G M and Winter H 1985 J. Phys. F: Met. Phys. 15 1337

[38] Hummler K and Fähnle M 1996 Phys. Rev. B 533272

[39] Bleaney B and Stevens K W H 1953 Rep. Prog. Phys. 16108

[40] Griffith J S 1961 The theory of transition-metal ions (Cambridge University Press)

[41] Newman D J and Ng B 1989 Rep. Prog. Phys. 52699

[42] Kuz'min M D and Tishin A M 2008 vol 17 (Elsevier B.V.) chap 3, p 149

[43] Tie-song Z, Han-min J, Guang-hua G, Xiu-feng H and Hong C 1991 Phys. Rev. B 438593

[44] Richter M, Steinbeck L, Nitzsche U, Oppeneer P M and Eschrig H 1995 J. Alloys Compd 225469

[45] Novák P 1996 Phys. Status Solidi B 198 729-740

[46] Steinbeck L, Richter M, Nitzsche U and Eschrig H 1996 Phys. Rev. B 537111

[47] Harashima Y, Terakura K, Kino H, Ishibashi S and Miyake T 2015 Proceedings of Computational Science Workshop 2014 (CSW2014) p 011021

[48] Novák P, Knížek K and Kuneš J 2013 Phys. Rev. B 87205139

[49] Buck S and Fähnle M 1997 J. Magn. Magn. Mater. 166297

[50] Blöchl P E 1994 Phys. Rev. B 5017953

[51] Giannozzi P et al. 2009 J. Phys. Condens. Matter 21395502

[52] Enkovaara J et al. 2010 J. Phys. Condens. Matter 22253202

[53] Kresse G and Joubert D 1999 Phys. Rev. B 591758

[54] Stevens K W H 1952 Proc. Phys. Soc. 65209

[55] Martin R M 2004 Electronic Structure: Basic theory and practical methods (Cambridge University Press)

[56] Information regarding the PAW datasets may be found at https://wiki.fysik.dtu.dk/gpaw/setups/setups.html

[57] Lebedev V and Laikov D 1999 Dokl. Math. 59477 
[58] Györffy B L and Stocks G M 1979 Electrons in Disordered Metals and at Metallic Surfaces (Springer US) chap 4, pp 89-192 Nato Science Series B

[59] Ebert H, Ködderitzsch D and Minár J 2011 Rep. Prog. Phys. 74096501

[60] Tamura E 1992 Phys. Rev. B 453271

[61] Bradley C J and Cracknell A P 1972 The Mathematical Theory of Symmetry in Solids (Oxford University Press)

[62] Däne M, Lüders M, Ernst A, Ködderitzsch D, Temmerman W M, Szotek Z and Hergert W 2009 J. Phys. Condens. Matter 21045604

[63] Larsen A H et al. 2017 J. Phys.: Condens. Matter 29273002

[64] Radwański R J 1987 J. Phys. F: Met. Phys. 17267

[65] Andreev A V 1995 Handbook of Magnetic Materials vol 8 (Elsevier North-Holland, New York) chap 2, p 59

[66] Herbst J F, Croat J J, Lee R W and Yelon W B 1982 J. Appl. Phys. 53 250-256

[67] Smidt F and Daane A 1963 J. Phys. Chem. Solids 24361

[68] Gabay A and Hadjipanayis G 2018 Scr. Mater. 154284

[69] Clark A E 1973 Appl. Phys. Lett. 23642

[70] Abbundi R and Clark A 1977 IEEE Trans. Magn. 131519

[71] Rhyne J J and Clark A E 1967 J. Appl. Phys 381379

[72] Ermolenko A 1976 IEEE Trans. Magn. 12992

[73] Buschow K, van Diepen A and de Wijn H 1974 Solid State Commun. 15903

[74] Klein H, Menth A and Perkins R 1975 Physica (Amsterdam) 80B+C 153

[75] Atzmony U, Dariel M P, Bauminger E R, Lebenbaum D, Nowik I and Ofer S 1973 Phys. Rev. B 74220

[76] Chikazumi S, Tanuma S, Oguro I, Ono F and Tajima K 1969 IEEE Trans. Magn. 5 265-268

[77] Ozaki T 2003 Phys. Rev. B 67155108

[78] Lejaeghere K et al. 2016 Science $\mathbf{3 5 1}$

[79] Patrick C E, Kumar S, Balakrishnan G, Edwards R S, Lees M R, Petit L and Staunton J B 2018 Phys. Rev. Lett. 120097202 